\title{
Yellow Substance in the Surface Waters of the Eastern Part of the Tropical Atlantic
}

\author{
V.I. Man'kovsky \\ Marine Hydrophysical Institute, Russian Academy of Sciences, Sevastopol, \\ Russian Federation \\ e-mail: emankovskaya@mail.ru
}

\begin{abstract}
The yellow substance (YS) content in the surface waters of the eastern part of the Atlantic Ocean tropical zone is studied. The investigations are carrie out on the polygon with coordinates $3^{\circ} \mathrm{S}-11$ ${ }^{\circ} \mathrm{N}$; and $19^{\circ}-22^{\circ} \mathrm{W}$. To define the YS content in the polygon waters, a new method is developed. Usually the YS content in water is assessed based on the value of its light absorption coefficient on the wavelength $390 \mathrm{~nm}$. It is defined, first, by measuring the seawater absorption coefficient. Second, for the surface waters, the phytoplankton pigments' absorption coefficient is subtracted from the seawater absorption coefficient; and for the deep-sea waters, the abundant, as compared to the seawater, absorption is attributed to yellow substance [1]. To define the YS content, the paper represents the method for measuring the beam attenuation coeffiient in the filtered seawater. In the suspension-free water the value of the beam attenuation coeffiient depends on light absorption by yellow substance and light attenuation by water the value of which for water is known. Thus, it is possible to define the YS absorption coefficient. For this purpose, to purify seawater of admixture, the filters with a pore diameter not exceeding $0.04 \mathrm{mcm}$ are to be used. The particles whose diameter is smaller than 0.04 mcm produce no influence upon the water optical characteristics. The investigation implies that the seawater is purufied by the filter the pore diameter of which is $0.4 \mathrm{mcm}$. In order to take into account the light attenuation coefficient of the particles that remained in the filtrare and the diameter of which is smaller than $0.4 \mathrm{mcm}$, a special method is developed. The spectral variability index of the YS light absoprtion coefficient is $\mu=0.0124 \mathrm{~nm}^{-1}$ in the waters of the region under investigation that is characteristic of mesotrophic waters. Application of the data from [6] on relation of the absorption coefficient of YS with its concentration permits to calculate the YS concentration in the polygon surface water. In the eastern part of the polygon the YS concentration exceeds the one in the western part that is explained by the fact that the currents from the upwelling zone on the Guinean shelf edge bring the waters with high YS concentration to the polygon. The YS contribution to the attenuation coefficient in the oceanic waters of different type being compared to the model simulations in [1], shows that, in general, the polygon waters are mainly similar to the coastal waters on the depths smaller than $100 \mathrm{~m}$.
\end{abstract}

Keywords: yellow substance, beam attenuation coefficient, suspended matter.

DOI: $10.22449 / 1573-160 X-2015-3-50-57$

(C) 2015, V.I. Man’kovsky

(C) 2015, Physical Oceanography

Introduction. Yellow substance (YS) in hydro-optics is the name of a part of the dissolved organic compounds that strongly absorb light in the spectrum shortwave band. At its high concentration, YS gives the water a yellowish color. Spectral dependence of the light absorption coefficient by YS is described by the exponential law $\kappa(\lambda)_{\mathrm{ys}} \sim \mathrm{e}^{-\mu \lambda}$ where $\lambda$ is the wavelength and $\mu$ is the coefficient conditioning the YS spectral variability.

YS isolation and determination of its concentration constitute a complicated problem; therefore such data are rather few in the oceanographic literature. In hydro-optics the YS content in the seawater is usually defined based on the value of the light absorption coefficient on the wavelength $390 \mathrm{~nm}$ [1, Ch. 6.3]. For seawater this coefficient is determined by subtracting the light absorption coefficient by phytoplankton pigments from the measured light absorption coefficient by seawater, and for deep sea waters - the surplus absorption (as compared to pure water) is attributed to the YS effect.

The coefficient of light absorption by YS can also be determined by measuring the beam attenuation coefficient (BAC) in the water purified of the suspended 
matter by filtration. In such water, in contrast to the unfiltered one, the BAC value depends only on the coefficient of light absorption by YS and the light absorption coefficient by seawater whose value is known.

In the models of marine suspension [2] it is assumed that the water optical characteristics are not influenced by the suspended matter with the particles whose radii are smaller than $0.02 \mathrm{mcm}$. In other words, water purification of suspension aimed at determining the YS content requires application of the filters with pore diameters $0.04 \mathrm{mcm}$.

In our experiment seawater was purified just by the filters with pore diameters 0.4 microns. These filters are widely used to determine the suspended matter concentration in water by the weighting method. The non-trapped particles whose radii are less than 0.2 microns produce practically no effect upon the suspended matter concentration in water. However, determination of the YS content requires taking into account influence of the particles that remained in the filtrate on the light attenuation coefficient. In this paper such a method is considered.

The region of work. The observations were carried out in the $52^{\text {nd }}$ cruise of $\mathrm{R} / \mathrm{V}$ "Mikhail Lomonosov" (January - March, 1990) on the polygon $\left(9^{\circ} \mathrm{N}-3{ }^{\circ} \mathrm{S}\right.$ and $19-22^{\circ} \mathrm{W}$ ). The polygon consisted of four sections along 19, 20, 21 and $22^{\circ} \mathrm{W}$. The stations were located on the sections every $0.5^{\circ}$.

Method of measurements. Water samples taken from the $3 \mathrm{~m}$ depth by a pump were strained through a filter (pore diameter is 0.4 microns). The unfiltered and filtered water were measured The spectral values of the beam attenuation coefficient $\varepsilon(\lambda)$ were measured both in unfiltered and filtered water. $\varepsilon(\lambda)$ was measured by a transparency meter [3] on the wavelengths 416, 432, 468 and $506 \mathrm{~nm}$. RMS measurement error was $0.01 \mathrm{~m}^{-1}(\ln )$.

During filtration and measurements of $\varepsilon(\lambda)$ in the filtered water the precautionary measures were observed to prevent the samples' contamination. The vessel (bottle, flask) in which the filtered water was collected was washed by preliminary distilled water, the cuvette in the transparency meter was also washed by distillate before measurements, and then - by filtered water.

Calculation method. The light attenuation coefficient in seawater $\varepsilon(\lambda)_{\mathrm{sw}}$ represents a sum of coefficients of: light attenuation by pure water $\varepsilon(\lambda)_{\mathrm{cw}}$, light scattering by large suspension $\sigma(\lambda)_{\text {large }}$ (organic particles with the radii $r>$ $>1$ micron), light scattering by fine suspension $\sigma(\lambda)_{\text {fine }}$ (mineral particles with radii $r<1$ micron), light absorption by yellow substance $\kappa(\lambda)_{\mathrm{ys}}$, light absorption by chlorophyll pig-ments $\kappa(\lambda)_{\text {chl }}[1$, Ch. 8.4]):

$$
\varepsilon(\lambda)_{\mathrm{sw}}=\varepsilon(\lambda)_{\mathrm{cw}}+\sigma(\lambda)_{\text {large }}+\sigma(\lambda)_{\text {fine }}+\kappa(\lambda)_{\mathrm{ys}}+\kappa(\lambda)_{\mathrm{chl}} .
$$

A filter with the pore diameter $0.4 \mathrm{mcm}$ permits to remove large suspension (plankton); chlorophyll pigments contained in the plankton; fine suspension with the particles' radii exceeding 0.2 microns. The light attenuation coefficient in the filtered water is as follows:

$$
\varepsilon(\lambda)_{\mathrm{fw}}=\varepsilon(\lambda)_{\mathrm{cw}}+\sigma(\lambda)_{r<0,2 \mathrm{mcm}}+\kappa(\lambda)_{\mathrm{yw}}
$$

To define the value of $\kappa(\lambda)_{\mathrm{yw}}$ by formula (2), the coefficients of light attenuation by pure water and light scattering by mineral suspension (the particles' radii are smaller than 0.2 microns) are to be subtracted from the measured light attenuation coefficient $\delta(\lambda)$ pure water and indicator mineral. 
The coefficient of light attenuation by pure water $\varepsilon(\lambda)_{\mathrm{cw}}=\kappa(\lambda)_{\mathrm{cw}}+\sigma(\lambda)_{\mathrm{cw}}$ was calculated using the data on the coefficient of light absorption by pure water $\kappa(\lambda)_{\mathrm{cw}}$ from [4] and the coefficient molecular scattering by pure water $\sigma(\lambda)_{\mathrm{cw}}$ from [1, Table. 6.1]. According to the results of calculations for the wavelengths 416, $432,468,506 \mathrm{~nm}$ the values of the attenuation coefficients $\varepsilon(\lambda)_{\mathrm{cw}}$ are $0.01 ; 0.0099$; 0.0137; $0.03 \mathrm{~m}^{-1}(\ln )$.

Correction for the mineral suspension light scattering was carried out in the following way. Relative contribution of scattering by mineral suspension (the particles' radii are smaller than 0.2 microns) to the total scattering by mineral suspension was calculated. At that the parameters of calculating the scattering by fine suspension from [1, Ch. 8.2] were used. Distribution of the particles' number $N(r)$ was assumed to be like Junge $r^{-v}$ with the following values of the distribution parameter $v . v=2.5$ for the particles with $r=0.01-0.05 \mathrm{mcm} ; v=3.5$ for the particles with $r=0.05-0.1 \mathrm{mcm} ; v=4.5$ for the particles with $r=0.1-1.3 \mathrm{mcm}$. The coefficient of light scattering by the particles whose radii vary from $r_{1}$ to $r_{2}$ was calculated by the formula

$$
\sigma=\pi \int_{r_{1}}^{r_{2}} K(\rho) N(r) r^{2} d r
$$

where $\rho=2 \pi r / \lambda$ is the diffraction parameter, $K(\rho)$ is the cross width of scattering by the particles. The values of $K(\rho)$ were taken from [3] for the particles with the refraction index $m=1.15$.

In the calculations the particles were divided into six groups with the following radii: $0.01-0.05 ; 0.05-0.1 ; 0.1-0.2 ; 0.2-0.5 ; 0.5-0.9 ; 0.9-$ $1.3 \mathrm{mcm}$. For each group, the particles' average radius was calculated by the formula

$$
<r>=\left(\frac{v-1}{v-2}\right)\left(\frac{r_{\max }^{2-v}-r_{\min }^{2-v}}{r_{\max }^{1-v}-r_{\min }^{1-v}}\right) .
$$

The number of particles $N(r)$ was calculated using the value $<\mathrm{r}>$ with regard to the distribution parameter $v$ for each range of the particles' radii. Relation $P=$ $=\sigma(0.01-0.2) / \sigma(0.01-1.3)$ of the coefficient of light scattering by the fine suspension particles (radii are $0.01-0.2 \mathrm{mcm}$ ) to the total coefficient of scattering by the mineral suspension particles (radii are $0.01-1.3 \mathrm{mcm}$ ) for the wavelengths 416, 432, 468, $506 \mathrm{~nm}$ used in the experiment, is equal to 0.134; $0.126 ; 0.111$; 0.107 , respectively.

Based on all the measurements (52 samples) carried out in the region of investigations, average spectral values of the light attenuation coefficient in the unfiltered and filtered water were calculated (Tabl. 1).

Table 1. Average spectral values of the light attenuation coefficient in the samples of unfiltered and filtered water

\begin{tabular}{l|l|l|l|l}
\hline Wave length, $\mathrm{nm}$ & 416 & 432 & 468 & 506 \\
\hline$<\varepsilon(\lambda)_{\mathrm{sw}}>, \mathrm{m}^{-1}(\ln )$ & 0.219 & 0.200 & 0.154 & 0.147 \\
$<\varepsilon(\lambda)_{\mathrm{fw}}>, \mathrm{m}^{-1}(\ln )$ & 0.120 & 0.106 & 0.067 & 0.069 \\
\hline
\end{tabular}

According to calculations in [1, Ch. 8.4], in the ocean waters $(H<100 \mathrm{~m})$ with the close to our case attenuation spectral index $\left\langle\varepsilon(416)_{s w}>=0.197 \mathrm{~m}^{-1}\right.$ (on our polygon $<\varepsilon(416)_{\mathrm{sw}}>=0.219 \mathrm{~m}^{-1}$ ), the mineral particles' contribution $F$ to the total 
light attenuation coefficient made up $22 \%$ in the spectral band 416 - $468 \mathrm{~nm}$ and $20 \%$ - on the wavelength $506 \mathrm{~nm}$.

Allowing for the values $F(\lambda)$ and the calculated coefficients $P$, the average values $\sigma(\lambda) r<0.2 \mathrm{mcm}$ are obtained in the following way :

$$
<\sigma(\lambda)_{r<0,2 \mathrm{mcm}}>=P(\lambda) F(\lambda)<\varepsilon(\lambda)_{\mathrm{sw}}>.
$$

Using theses data the values of $<\kappa(\lambda)_{\mathrm{ys}}>$ are calculated:

$$
<\kappa(\lambda)_{\mathrm{ys}}>=<\varepsilon(\lambda)_{\mathrm{fw}}>-\varepsilon(\lambda)_{\mathrm{cw}}-<\sigma(\lambda)_{\mathrm{r}<0,2 \mathrm{mcm}}>.
$$

The values $<\sigma(\lambda)_{r<0,2 \mathrm{mcm}}>$ and $<\kappa(\lambda)_{\mathrm{ys}}>$ are represented in Tabl. 2 .

Table 2. Spectral values of the coefficients of light scattering by mineral suspension (particles' radii are smaller than $0.2 \mathrm{mcm}$ and light absorption by YS

\begin{tabular}{l|l|l|l|l}
\hline Wavelength, $\mathrm{nm}$ & \multicolumn{1}{c|}{416} & \multicolumn{1}{c}{432} & \multicolumn{1}{c}{468} & \multicolumn{1}{c}{506} \\
\hline$<\sigma(\lambda)_{r<0,2 \mathrm{mcm}^{>}}, \mathrm{m}^{-1}(\ln )$ & 0.0065 & 0.0055 & 0.0038 & 0.0032 \\
$<\kappa(\lambda)_{\mathrm{vs}}>, \mathrm{m}^{1}(\ln )$ & 0.104 & 0.09 & 0.049 & 0.036 \\
\hline
\end{tabular}

Fig. 1 shows the graph of the values $<\kappa(\lambda)_{\mathrm{ys}}>$ spectral variability. The exponent $\mu$ coefficient turned out to be equal to $0.0124 \mathrm{~nm}^{-1}$. In [1, Tabl. 6.4] the value $\mu=0.012$ is given for the ocean surface waters of a mesotrophic type. The observations carried out by biologists in the $47^{\text {th }}$ cruise of $\mathrm{R} / \mathrm{V}$ "Mikhail Lomonosov" [5] show that the water of the eastern Tropical Atlantic are attributed to a mesotrophic type. Thus, the obtained data on the coefficient $\mu$ in the polygon waters are in agreement with the data of [1, Tabl. 6.4].

To estimate the discrete values $\kappa(\lambda)_{\mathrm{ys}}$, the coefficient $\langle S(\lambda)\rangle=\left\langle\kappa(\lambda)_{\mathrm{ys}}\right\rangle /$ $\left[<\varepsilon(\lambda)_{\mathrm{fw}}>-\varepsilon(\lambda)_{\mathrm{cw}}\right]$ equal to $0.942 ; 0.940 ; 0.929$ and 0.919 for the wavelengths 416 , 432, 468 and $506 \mathrm{~nm}$, respectively, was calculated from the averaged data. The values $\left[\varepsilon(\lambda)_{\mathrm{fw}}-\varepsilon(\lambda)_{\mathrm{cw}}\right]$ were multiplied by this factor. When the coefficient $\langle S(\lambda)>$ was used, the factor $F$ characterizing relative contribution of fine suspension to the coefficient of light attenuation by sea water was assumed unchangeable in the polygon waters. It was confirmed by the modeling data in [1, Ch. 8.4] according to which the factor $\mathrm{F}$ in the ocean waters $(H<100 \mathrm{~m})$ changes insignificantly: in the open ocean $\left(<\varepsilon(416)_{\mathrm{sw}}>=0.197 \mathrm{~m}^{-1}\right)$ the $F$ contribution of mineral particles to the total light attenuation coefficient was $22 \%$ in the spectral band $416-468 \mathrm{~nm}$ and $20 \%$ on the wavelength $506 \mathrm{~nm}$; in the coastal ocean waters $\left(<\varepsilon(416)_{\mathrm{sw}}>=0.79 \mathrm{~m}^{-1}\right)$ it was $17 \%$ in the spectral band $416-432 \mathrm{~nm}$ and $16 \%$ - in the band $468-506 \mathrm{~nm}$. The range of the light attenuation coefficient variation on the polygon $\delta(416)_{\mathrm{sw}}=0.14-0.29 \mathrm{~m}^{-1}$. In other words, possible changes of the factor $F$ on the polygon could slightly influence determination of the values $\kappa(\lambda)_{\mathrm{ys}}$.

Specific index of light absorption by YS on the wavelength $450 \mathrm{~nm}$ $\kappa(450)_{\text {ud. ys }}=0.212 \mathrm{~m}^{2} \cdot \mathrm{g}^{-1}$ is calculated in [6] that permits to determine the YS concentration in water by measuring $\kappa(450)_{\text {ud. ys }}$ in the following way: $C_{\mathrm{ys}}, \mathrm{g} \cdot \mathrm{m}^{-3}=$ $=\kappa(450)_{\mathrm{ys}} / \kappa(450)_{\mathrm{ud} . \mathrm{ys}}$. We have found no other data on the value of $\kappa(\lambda)_{\mathrm{ud} . \text { ys }}$ in the ocean waters in scientific literature. In this connection the following question arises: to what extent is the value $\kappa(450)_{\text {ud. ys }}$ given in [6] universal. However, in view of the foresaid, it was decided to consider the YS distribution on the polygon using the values of its concentration calculated using $\kappa(450)_{\text {ud. ys }}$ from [6]. 


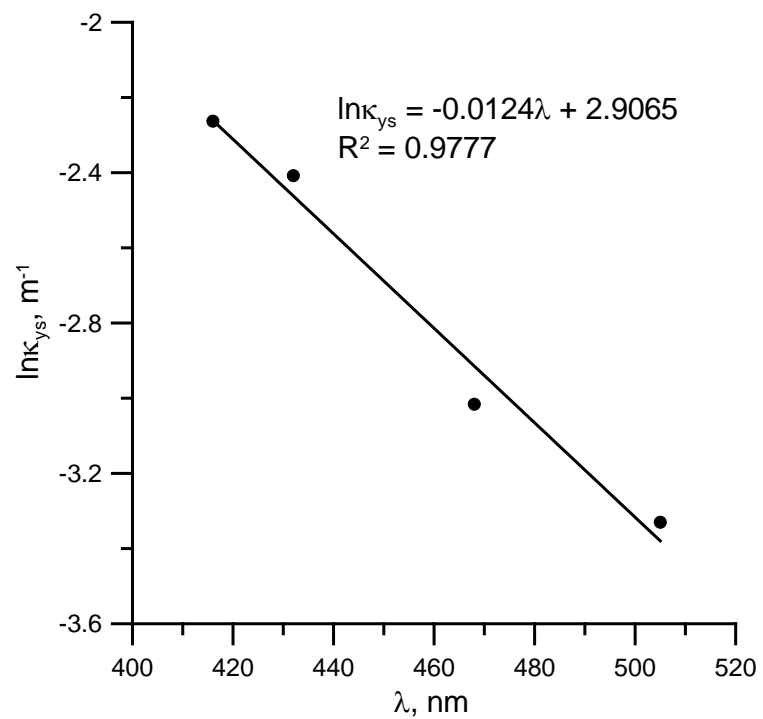

Fig. 1. Spectral variability of the coefficient of light absorption by yellow substance in the surface waters of the region ( $R^{2}$ is the determination coefficient)

To define the YS concentration, the measurement data on the coefficient of light attenuation in filter water on the wavelength $468 \mathrm{~nm}$ were used. The value of $C_{\text {ys }}$ was calculated by the formula

$$
C_{\mathrm{ys}}, \mathrm{g} \cdot \mathrm{m}^{-3}=\left[\varepsilon(468)_{\mathrm{fw}}-\varepsilon(468)_{\mathrm{cw}}\right]<S(468)>\exp \frac{[-0,0124(450-468)]}{\kappa(450)_{\mathrm{ud} . \mathrm{ys}}} .
$$

Results of calculations and discussion. Fig. 2 shows the YS distribution concentration on the polygon. The range of $C_{\mathrm{ys}}$ variability was $0.076-0.403 \mathrm{~g} \mathrm{~m}^{-3}$. The average value of the YS concentration in the polygon waters $\left\langle C_{\mathrm{ys}}\right\rangle_{19-22}=$ $=0.294 \mathrm{~g} \cdot \mathrm{m}^{-3}$.

The YS concentration on the polygon increased from its eastern part towards the eastern one. In the western part on the sections along 21 and $22{ }^{\circ} \mathrm{W}$ the average value of the YS concentration was $\left\langle C_{\mathrm{ys}}\right\rangle_{21-22}=0.256 \mathrm{~g} \cdot \mathrm{m}^{-3}$ and in the eastern part on the sections along 19 and $20^{\circ} \mathrm{W}-\left\langle C_{\mathrm{ys}}\right\rangle_{19-20}=0.311 \mathrm{~g} \cdot \mathrm{m}^{-3}$. Such distribution of $C_{\mathrm{ys}}$ is explained by the waters with high YS concentration coming from the upwelling zone on the periphery of the Guinean shelf to the polygon. Based on the measurements in the upwelling zone at $9^{\circ} 17^{\prime} \mathrm{N}$ and $13^{\circ} 50^{\prime} \mathrm{W}$ the $\mathrm{YS}$ concentration constituted $0.90 \mathrm{~g} \cdot \mathrm{m}^{-3}$.

Fig. 3 represents the currents in the polygon surface layer calculated by the dynamic method. Comparison of the currents' chart with Fig. 2 shows presence of the western currents (i. e. moving from the upwelling region on the Guinean shelf) in the polygon eastern part where the YS concentration is high.

Yellow substance enters the ocean in two ways: it is brought from the land with the river waters and is formed directly in the ocean as a result of chemical decomposition of dead plankton organisms. The first way of the YS formation is significant only for the coastal waters and in the ocean open regions the autochthonous YS dominates. That is why, in the ocean open areas the correlation between the YS and suspension concentrations should be observed since the 
suspension basic part consists of live phytoplankton and dead plankton in a form of detritus.

In the water samples taken on the polygon the suspended matter concentration was defined by the weighting method simultaneously with determining the YS one. The correlation between the YS and suspended matter concentrations was calculated. The correlation coefficient calculated based on the data for the whole polygon turned out to be low: $R=0.212$. It is explained by presence of waters from the area of coastal upwelling in the polygon eastern part. The YS-suspension relation in these waters is different from that in the other areas of the polygon.

Based on the foresaid the correlation between the YS and suspended matter concentrations was calculated for the polygon regions where, according to the map (Fig. 3), no water flows from the coastal upwelling are observed. The following polygon regions were chosen on 21 and $22{ }^{\circ} \mathrm{W}$ : in the southern part - from $3{ }^{\circ} \mathrm{S}$ to $0^{\circ}$, in the northern part - from 3 to $8^{\circ} \mathrm{N}$. The correlation coefficient constituted $\mathrm{R}=$ $=0.697$. Relation between YS and suspension in this case is shown in Fig. 4.

The data on YS obtained on the polygon were compared with those from [1, Ch. 8.4] which represents the results of calculating spectral contribution of light absorption by YS to the beam attenuation coefficient in the ocean waters. The values of $\kappa(450)_{\mathrm{ys}}$ were used for comparison. Tabl. 3 contains the values of $\kappa(450)_{\mathrm{ys}}$ on the polygon and in the ocean waters of different types from [1, Ch. 8.4].
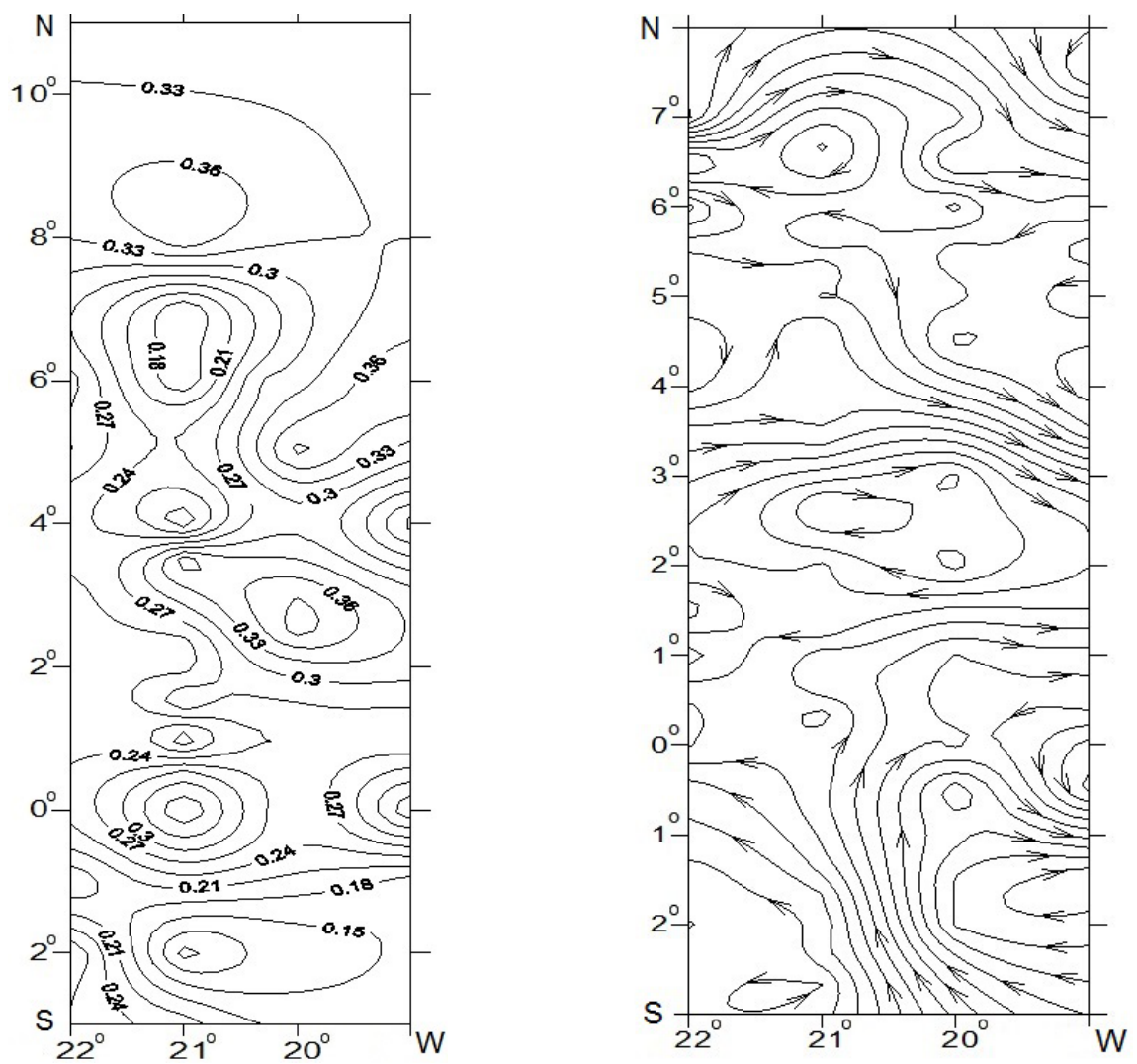

Fig. 2. Distribution of the yellow sub- Fig. 3. Map of the currents in the polygon surface waters stance concentration in the polygon surface waters $\left(\mathrm{g} \cdot \mathrm{m}^{-3}\right)$ 




Fig. 4. Relation between the yellow substance and suspended matter concentrations in the polygon areas free of the coastal upwelling waters

Table 3. Coefficient of light absorption by YS $\kappa(450)_{\mathrm{ys}}$ in the polygon and ocean waters of different types from [1, Ch. 8.4]

\begin{tabular}{lcccc}
\hline \multicolumn{1}{c|}{ Waters (region) } & $\begin{array}{c}\text { Number of } \\
\text { samples }\end{array}$ & $\begin{array}{c}\left\langle C_{\text {хл }}>,\right. \\
\mathrm{mg} \cdot \mathrm{m}^{-3}\end{array}$ & $<\kappa(450)_{\text {жв }}>, \mathrm{m}^{-1}$ \\
\hline polygon & 51 & - & 0.054 \\
$\begin{array}{l}\text { eastern part of the polygon } \\
\left(19 \text { and } 20^{\circ} \mathrm{W}\right)\end{array}$ & 20 & - & 0.066 \\
$\begin{array}{l}\text { zones of Guinean upwelling } \\
\left(9^{\circ} 17^{\prime} \mathrm{N} ; 3^{\circ} 50^{\prime} \mathrm{W}\right)\end{array}$ & 1 & - & 0.191 \\
$\begin{array}{l}\text { oceanic }(H<100 \mathrm{~m}) \\
\text { coastal }(H<100 \mathrm{~m})\end{array}$ & 49 & 0,027 & 0.020 \\
$\begin{array}{l}\text { nearby the Arabian Peninsula } \\
(H=10 \mathrm{~m})\end{array}$ & 23 & 0,51 & 0.072 \\
\hline
\end{tabular}

The comparison shows that the values of the coefficient of light absorption YS for the whole polygon $\left(<\kappa(450)_{\mathrm{ys}}>_{19-22}=0.054 \mathrm{~m}^{-1}\right)$ and especially its eastern part $\left(<\kappa(450)_{\mathrm{ys}}>_{19-20}=0.066 \mathrm{~m}^{-1}\right)$ are similar to the coefficient values for the coastal waters $(H<100 \mathrm{~m})$. In the southern part of the polygon on 21 and $22{ }^{\circ} \mathrm{W}$ the values $\kappa(450)_{\mathrm{ys}}=0.016 \mathrm{~m}^{-1}$ and $\kappa(450)_{\mathrm{ys}}=0.032 \mathrm{~m}^{-1}$ were observed, respectively. They are of the same order as those in the ocean waters $(\mathrm{H}<100 \mathrm{~m})$. In the zone of the Guinean upwelling the value $\kappa(450)_{\mathrm{ys}}=0.191 \mathrm{~m}^{-1}$ is high as well as near the Arabian Peninsula $\left(\kappa(450)_{\mathrm{ys}}=0,146 \mathrm{~m}^{-1}\right)$.

\section{Conclusions.}

1. The method for determining spectral coefficients of light absorption by YS in the ocean waters is developed based on application of the beam attenuation coefficient measured in the shortwave spectrum band $(416-506 \mathrm{~nm})$ in the water samples filtered through a filter with a pore diameter $0.4 \mathrm{mcm}$. 
2. Spectral coefficients of light absorption by YS in the surface waters (depth $3 \mathrm{~m}$ ) on the polygon located in the eastern part of the Tropical Atlantic are defined.

3. The spectral variability index of the coefficient of light absorption by YS in the polygon waters $\mu=0.0124$ is calculated.

4. The YS concentration in the polygon waters is calculated. In the eastern part of the polygon the YS concentration is higher than in the western one. It is due to the waters with high concentration of YS brought to the polygon by the flows from the upwelling zone on the Guinean shelf periphery.

5 . The YS and suspended matter concentrations are compared. In general the correlation coefficient over the whole polygon is insignificant $(R=0.212)$ that is a result of influence of the waters brought to the polygon from the coastal upwelling zone. In the polygon zones free of such waters the correlation coefficient is high $(R=0.697)$.

6. Comparison of the values $\kappa(450)_{\mathrm{ys}}$ in the polygon waters with the results of model calculations of the YS contribution to the light attenuation coefficient in the ocean waters of different types [1] showed that on the whole the polygon waters are similar to the coastal waters on the depths less than $100 \mathrm{~m}$. At the same time in some parts of the polygon the values $\kappa(450)_{\mathrm{ys}}$ characteristic of the oceanic waters were observed.

\section{REFERENCES}

1. 1983, “Optika okeana. T. 1. Fizicheskaya optika okeana [Ocean optics. Vol. 1. Physical optics of the ocean]”, Ed: A.S. Monin, Moscow, Nauka, 371 p. (in Russian).

2. $\quad$ Shifrin, K.S., Salganik, I.N., 1973, “Tablitsy po svetorasseyaniyu. T. 5. Rasseyanie sveta modelyami morskoy vody [Tables on light scattering. Vol. 5 Light scattering by seawater models]”, Leningrad, Gidrometeoizdat, 218 p.

3. Man’kovsky, V.I., 2012, “Spektralny laboratorny prozrachnomer s peremennoy bazoy [Spectral laboratory transparency meter with a variable basis]”, Sistemy kontrolya okruzhayushchey sredy, pp. 56-60 (in Russian).

4. Pope, R.M., Fry, E.S., 1997, “Absorption spectrum (380-700 nm) of pure water. II. Integration cavity measurements”, Appl. Optics, vol. 36, no 33, pp. 8710-8723.

5. Senichkinf, L.G., Georgieva, L.V., 1987, "Fitoplankton vostochnoy chasti Tropicheskoy Atlantiki [Phytoplankton of the eastern part of the Tropical Atlantic]”, Okeanologicheskie issledovfniya v vostochnoy chasti Atlanticheskoy tropicheskoy energoaktivnoy zony, Materialy 47-go reisa NIS “Mikhail Lomonosov", Sevastopol, MGI AN USSR, pp. 125-149 (in Russian).

6. Nyquist, G., 1979, "Investigation of some optical properties of sea water with special reference to lignin sulfonates and humic substances”, Thes. Dep. Anal. Mar. Chem., Gotheburg, 200 p. 